\title{
FACTORES QUE INFLUYEN PARA LA SUCESIÓN EXITOSA DE LAS EMPRESAS FAMILIARES EN CIUdAd JUÁREZ
}

\author{
Ricardo Carrillo Vega \\ Leslie Sujey González Lara \\ Jesús Adolfo García Ortega \\ Javier Olaf Sánchez Pérez \\ Universidad Autónoma de
}

Ciudad Juárez

Recibido: 25 de octubre de 2018 Aceptado: 8 de febrero de 2019

\section{RESUMEN}

A ctualmente en México hay pocas empresas racionales, ya que solo el $35 \%$ de ellas logra sobrevivir a la segunda generación, y únicamente el $13 \%$ llega a la tercera generación (Álvarez, 2014). La presente investigación tiene como objetivo principal identificar los factores que influyen en el éxito de empresas familiares que logran sobrevivir a la tercera generación después de la sucesión. Se trata de un estudio descriptivo, transversal y con enfoque cuantitativo, con una muestra representada por 11 empresas familiares de
Ciudad Juárez, Chih. Se pudo observar que aspectos como el liderazgo, el sentido de pertenencia y una buena planeación a largo plazo son factores que influyen para que las empresas familiares puedan subsistir.

Palabras clave: Empresa familiar, sucesión.

\section{Abstract \\ urrently in Mexico there aren't many transgenerational fa-} mily businesses, only $35 \%$ of them, survive the second generation, and only $13 \%$ reach the third generation (Álvarez, 2014). The objective of this 
research is to identify the factors that influence the success of family business manage to reach the third generation after the succession. It is a descriptive, cross-sectional study, with a quantitative approach, with 11 family business sample from Ciudad Juarez. Aspects such as leadership, sense of belonging, long-term plans as a factors of family business success were observed.

Keywords: Family business and succession.

\section{REVISIÓN DE LITERATURA} Empresas familiares

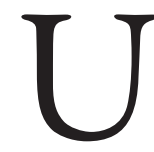

na empresa es el conjunto de personas que trabaja de manera cooperativa y sostenida en el tiempo, es decir, continuamente, pues sus integrantes saben que de esta manera llegarán con un mayor resultado a los objetivos que tienen en común (Audisio, 2014).

Romero (2013) define empresa familiar como una organización que es la propiedad de una familia; por su parte, Belausteguigoitia (2005) menciona que las empresas familiares son organizaciones operadas y controladas por una familia, adicionalmente, señala que son empresas con alma, con espíritu, ya que considera que el corazón de las familias está en ella. De igual manera, refiere que puede ser complicada su administración puesto que generalmente son controladas por una familia, pero no operadas por los miembros de esta.

Razones de la creación de empresas familiares

7 xisten diversas razones 4 por las cuales los fundadores o líderes de las empresas familiares desean una continuidad de su empresa. Gallo \& Amat (2003) destacan, por una parte, la oportunidad para los hijos a fin de que tengan una posibilidad de crecimiento personal, ejercer más control sobre su propio futuro, así como la oportunidad de una posibilidad de independencia. Por otra parte, conservar la herencia y el legado familiar, lo que puede ser para mantener o consolidar una tradición, una historia o sus raíces o simplemente para generar algo que permanezca en el tiempo, al igual que man- tener unida a la familia de tal forma que trabaje junta y se fortalezcan los lazos, o ya sea para pasar más tiempo con los hijos. Por otra parte, crear ventajas económicas, riquezas y garantizar la riqueza económica de la empresa, y de esa forma asegurar el mantenimiento de los ingresos y de proyectos personales tras su retirada.

Modelo de los 3 círculos

7 agiuru y Davis (1982) proponen un mo-

1 delo denominado "modelo de los 3 círculos", en donde destacan que la empresa familiar está regida por la interacción de tres aspectos: la familia, la propiedad y la empresa. En el círculo de la familia se hace referencia a los lazos sanguíneos, en el círculo de la propiedad, a los accionistas, y finalmente en el círculo de la empresa, a las funciones desempeñadas dentro de esta. Estos círculos al interactuar dan origen a otros "mini círculos", los cuales explican como se muestra a continuación y detalla en la figura 1: familia, propiedad y gestión (7): miembros de la familia que poseen acciones y ocupan algún puesto dentro de la direc- 
ción de la empresa; familia y propiedad (4): miembros de la familia que tienen un determinado número de acciones de la empresa; familia y gestión (6): miembros que participan en la empresa y desarrollan labores en ella, pero no poseen alguna acción; propiedad y gestión (5): individuos que participan en la empresa y desarrollan labores de dirección, sin pertenecer a la familia que ostenta el control de la organización.

\section{Planeación EN LA SUCESIÓN EXITOSA}

$\left\lceil\begin{array}{l}\text { no de los mayores } \\ \text { retos a los que la em- } \\ \text { presa familiar se en- }\end{array}\right.$ frentará, es la sucesión, situación por la que si no se tiene un plan bien definido sobre cómo será este proceso, existe una gran posibilidad de que la empresa no sobreviva a esta etapa (Modest, 2000).

Escobar (2014) menciona que la planeación sucesoria busca satisfacer 4 tipos de finalidades: las interpersonales, judiciales, económicas y fiscales. La interpersonal busca evitar conflictos en el seno familiar, pues si se hace de la manera correcta y en el tiempo debi-

Figura 1. Modelo de los 3 círculos

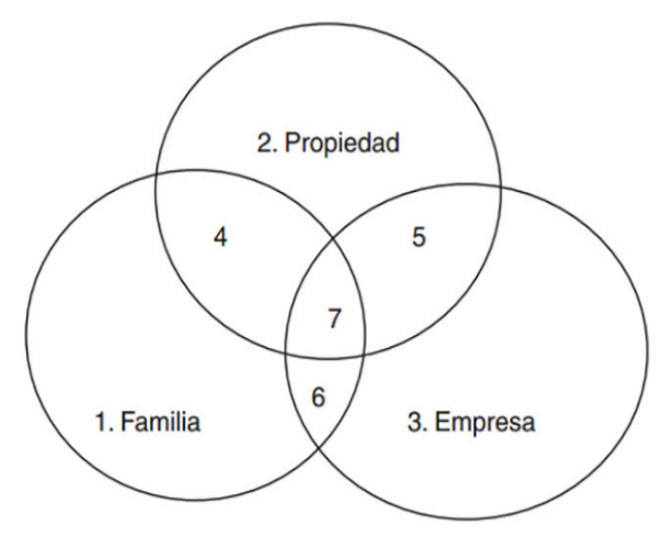

Fuente: Arenas Cardona \& Rico Balvin (2014).

do, todos los pormenores de lo que sucederá en la empresa quedarán resueltos bajo el consenso de los interesados.

Camacho (2012) señala que es importante que exista voluntad del empresario para que los acuerdos y la reglamentación del proceso sucesorio se haga por escrito y quede asentado en un documento legal formalmente constituido (protocolo familiar), debido a que un alto índice de cierre de empresas familiares se da debido a que no existía un consenso sobre quién siente el derecho de ocupar el máximo puesto de la organización y esto se refleja en conflictos hacia el interior de la familia, con efectos devastadores, tales como discriminación de género (no sería capaz de dirigir la empresa por ser hombre o mujer, yo como hombre merezco más que ella, el puesto requiere de un hombre, etcétera), división de funciones en el interior y exterior de la empresa, formación de grupos de trabajo y de poder antagónicos (no se tiene el mismo objetivo organizacional), presencia de envidias, desconfianza, golpes bajos, habladurías, trato inapropiado a clientes y proveedores, y a su vez esto repercutiría en el desánimo del personal de la empresa para realizar un mejor trabajo y dar un esfuerzo mayor. 
Para el buen funcionamiento del plan de sucesión se deberá contar con un calendario en donde se especifiquen los pasos y etapas a seguir, tomando en cuenta que cada persona, empresario, familia, es diferente y no puede existir un número o cantidad de tiempo estimado, dependiendo del tipo de control manejado en cada empresa (Modest, 2000).

Debe cumplirse con ciertas fases según Modest (2000), para hacer más ligero el cambio en el poder, teniendo fechas establecidas y conocidas por cada uno de los miembros de la empresa que pudieran verse afectados en alguna forma por la sucesión, no solo familiares sino también personas con intereses directos a la organización (empleados, clientes, proveedores, accionistas) y creando así certidumbre al entorno tanto interno como externo de la empresa.

\section{Etapas de la sucesión}

Modest (2012) señala que un buen plan de sucesión consta de 4 etapas, ordenadas cronológicamente cada una de ellas con una importancia especial para cada involucrado en el proceso. El buen manejo y en- tendimiento de estas será sin duda un gran paso para el éxito de la sucesión:

1.- Planificación de la sucesión. En esta etapa la familia es la encargada de reconocer la necesidad de un sucesor en determinado momento y basar la planeación en la percepción de todos los involucrados y tomar las decisiones más convenientes para ellos, teniendo en cuenta que esto no será fácil, pero es necesario.

2.- Preparación del sucesor. Esta es la etapa que suele ser la más larga del proceso, teniendo en cuenta cuál formación será la adecuada y necesaria para la empresa; de igual manera, buscar el apoyo de todas las personas involucradas en el negocio, para que esto logre generar un aprendizaje de todos los aspectos de la empresa y se logre familiarizar con la cultura y objetivos de esta, en caso de que no lo esté. Siendo esta etapa en la cual el sucesor tiene que mostrar capacidades para darle continuidad al negocio, y si no es apto, hacer los cambios pertinentes. Press (2011) considera que la preparación consiste en formar profesionalmente a los miembros de la familia que tengan como prioridad trabajar en la empresa y también que se sientan aptos para hacerlo, así como también a la pregunta de cuándo empezar esta preparación tan pronto como se pueda, con la intención de tener una preparación mucho más completa.

3.- Toma de posesión de la empresa de manera formal. En este momento ya sería muy difícil realizar algún cambio repentino en la decisión tomada, porque esto significaría años de trabajo y planeación desperdiciados, representaría reiniciar el proceso para encontrar a la persona indicada capaz de tomar las riendas de la organización. No significa que se tenga que ceder la empresa en su totalidad; también deberá ser un proceso de control planeado, pero dejando que sea la imagen principal de la empresa.

4.- Control total de la empresa. Esta última etapa es 
la que cierra el círculo de la sucesión; aquí el sucesor toma el control total de la empresa; sin embargo, el sucedido jugará un papel muy importante, ya que la retirada, independientemente del tiempo que estuvo a cargo de la empresa, no es fácil. El relevado requerirá del apoyo total de la familia para hacer este trance lo más ligero posible y también para que él vea que sigue siendo parte fundamental dentro del negocio, tal vez ya no de una manera directa, pero sí en el aspecto de ser un solucionador de contingencias y un consejero para el nuevo líder de la organización. Víctor \& Jago (1990) mencionan que se debe llevar a cabo una serie de planes, pensando en las necesidades no solo de la empresa, sino de todos los que de alguna forma puedan verse afectados por la sucesión

\section{LIDERAZGO EN} LA SUCESIÓN EXITOSA

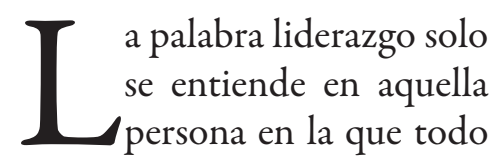

su actuar está basado en una motivación trascendente, en el aprendizaje positivo de los demás (Gómez, 2016); se centra en tratar de unir a todos los integrantes de la organización, en encontrar un equilibrio entre los clientes, proveedores, comunidad, empleados, accionistas, gobierno y medio ambiente. Un buen líder es el que basa su organización en ayudar a todos los partícipes de ella, y esto a su vez le llevará a obtener los resultados económicos deseados y necesarios para la supervivencia de la misma a largo plazo.

Gómez (2016) menciona que existen tres generaciones de liderazgo en la empresa familiar: la primera y fundadora de la organización se maneja de una manera más apegada a sus trabajadores; generalmente el dueño o fundador de la empresa es quien toma las decisiones sin necesidad de contar con consejos de administración o ejecutivos; la asignación de tareas tampoco es un factor determinante para el funcionamiento de la empresa porque es el dueño quien hace el papel de propiedad, gobierno, dirección y operación.
Los líderes de segunda generación son jóvenes preparados con una carrera profesional, debiendo ellos adaptarse a la innovación y uso de tecnologías de información en una economía abierta, con clientes cada vez más exigentes en cuanto a calidad y satisfacción de sus necesidades, por lo que el líder tiene que aprender a confiar en los demás, a delegar responsabilidades, a trabajar en equipo, en fin, a desarrollar habilidades muy diferentes a las que utiliza el fundador de la organización.

La tercera generación debe, aparte de desarrollar las habilidades antes mencionadas, tener la capacidad de relacionarse entre los socios, buscando con esto el crecimiento de la empresa y la posibilidad de ofrecer nuevos productos y servicios, por lo que deben ser no solo inteligentes con los empleados, sino con todos los integrantes de la organización.

Fiedler (1967) con su modelo de liderazgo contingente menciona que el tipo de liderazgo se definirá de acuerdo con tres dimensiones: 1) el grado de estructuración presente en el trabajo de grupo; 2) la cantidad de poder con que 
contaba el líder según la posición que ocupaba en la empresa, y 3) la calidad de las relaciones interpersonales entre el líder y los demás miembros del grupo. Por su parte, Hersey \& Blanchard (1982) distinguen cuatro estilos de liderazgo (el que habla, el que vende, el que participa y el que delega), considerando que cada uno de ellos es válido para diferentes situaciones en la empresa, diferenciando al líder del modelo de Fiedler y dándole más peso por lo que hace y no por lo que es.

\section{Perfil del lider}

Es importante definir el perfil del líder que será el sucesor de la empresa, pues debe ser el ideal para el cargo, pero se debe tomar en cuenta que los atributos y/o condiciones deben adaptarse según las necesidades de la empresa, pues las condiciones de los negocios cambian y se requiere que los directivos tengan habilidades distintas. Para ello Martínez (2011) menciona algunos atributos y condiciones deseados: que se gane el respeto de la familia y de todos los involucrados; que siempre haya sido una alternativa para la sucesión; que tenga una visión clara de cómo competir en un mundo tan globalizado, que concuerde con las estrategias de la empresa; que tenga la formación empresarial adecuada (experiencia y estudios); que sienta un gran sentido de pertenencia hacia la empresa; que tenga su propio y leal equipo de ejecutivos de confianza; que realmente quiera dirigir la empresa y se sienta orgulloso de ser el líder.

\section{Preparación del lider candidato}

Todo líder debe tener una formación y más si es este líder es el que asumirá la sucesión de una empresa familiar que quiere perdurar, por eso se requieren 3 fases: en la primera se muestra una formación académica, para después darle paso a la etapa de la experiencia laboral externa y finalmente a una de trabajo interno antes de asumir el cargo de la empresa (Martínez, 2011).

Martínez (2011) menciona que el sucesor debe de adquirir poder y autoridad, él nos hace una diferencia entre poder y autoridad, definiendo poder como la capacidad de influir sobre el comportamiento de otra persona y a la autoridad como el derecho de hacerlo y esto es considerado como la base del liderazgo.

\section{Sentido de pertenencia} y compromiso

Otro de los aspectos importantes para el logro exitoso de la sucesión es el sentido de pertenencia y compromiso del líder. Quijando, Magaña y Pérez (2011) destacan cuatro bases para ese compromiso: 1) compromiso afectivo apoyado en un lazo emocional, caracterizado por la confianza y consecución de los objetivos mutuos, basándose en valores compartidos, los cuales normalmente se dan cuando el sucesor se identifica plenamente con los objetivos y valores planeados en la empresa (Folle, 2015), 2) compromiso normativo basado en la percepción del sentido de obligación y el deber, en donde tiene más peso el sentimiento de no defraudar a la familia (Folle, 2015) y a quien depositó la confianza en él, 3) compromiso calculador que considera los costos de oportunidad involucrados y la forma de evitarlos o disminuirlos y, 4) compromiso imperativo, que 
comprende la percepción de las necesidades y la dependencia hacia la organización como único recurso de acción disponible para el sucesor.

Gallo \& Amat (2006) mencionan algunos factores que influyen en el compromiso en la empresa: el liderazgo carismático, generador de seguridad e ilusión entre los miembros de la familia; un proyecto empresarial basado en una planeación ambiciosa y en donde los miembros de la familia puedan implicarse; así como también los valores familiares y empresariales adquiridos a través de acciones hechas por otros miembros de la familia de generaciones pasadas.

Por su parte, Quijano, Magaña \& Pérez Canul (2011) mencionan que el grado de compromiso del sucesor será beneficiado significativamente según sus conocimientos sobre los antecedentes de la empresa, generalmente adquiridos a través de la comunicación en el hogar, la relación que en determinado momento llegara a tener con los miembros de la organización, tales como familia, clientes, proveedores, empleados y en la forma en que este maneje determinados conflictos que se den en el entorno familia-trabajo.

Lambrech y Astrachan (1994) subrayan que la relación familiar juega un papel muy importante y que esta es medida por dos componentes: el compromiso y la calidad. El compromiso familiar es con la empresa; cuando un propietario involucra a la familia, pues en esta se van valores, identidad, cultura y tradiciones familiares, con lo cual se intenta asegurar la longevidad de la empresa. La calidad es sobre la relación entre dueño-administrador y el sucesor, pues una buena relación refleja confianza, ayuda mutua, buena comunicación y se adquieren nuevos conocimientos.

\section{Valores dentro del liderazgo}

Uno de los aspectos que llama la atención de los fundadores como creadores de la empresa es como ellos crean una estructura social y empresarial, es por ello que los fundadores de las empresas plasman sus valores en el ámbito de la organización (García, 2001). García (2001) menciona que la organización viene marcada por un compromiso de valores, es decir, se hacen elecciones para tomar las decisiones sobre la naturaleza de la empresa, sus objetivos, métodos y papeles a desempeñar, siendo un líder de esa empresa un experto en la promoción y protección de valores.

En la familia es muy importante inculcar y transmitir los valores de generación en generación. Existen diversas definiciones, pero Martínez (2011) dice que son los rectores de la acción que los miembros de la familia no pueden negociar, pues a estos no se pueden renunciar. Martínez (2011) nos dice que ha trabajado con empresas familiares de distintos países y él ha detectado que existen algunos valores que se repiten con frecuencia. Mostrándose los 10 valores que tuvieron más frecuencia en una muestra de 32 familias empresarias de México, Argentina, Brasil, Chile y Perú. Con 24 menciones se encuentran la honestidad, honradez, integridad y trasparencia; con 18 menciones la unidad familiar y la lealtad; el respeto con 16 menciones; la solidaridad, así como la humildad, sencillez, sobriedad y austeridad (cuatro valores como uno solo) con 10 
menciones; la justicia y equidad con 9; con 8 menciones la generosidad; seriedad y responsabilidad con 7 menciones; y el compromiso y dedicación al trabajo con 6 .

El refuerzo de los valores aprendidos en el seno familiar es un factor clave para el desarrollo y fortalecimiento de una empresa familiar (Guerrero, 2006), la ausencia de estos valores se transmitirá a toda la empresa y afectará el sentido ético del trabajo y las relaciones entre los miembros de la organización.

\section{Consejo familiar}

Folle (2015) señala que existe algo denominado "consejo familiar” y que este es el que contribuye a que se mantengan los valores, la cultura y la tradición de la empresa familiar. El consejo suele limitarse solo a tratar aspectos relacionados con la familia, la transmisión de principios y los valores que guiarán a la empresa y así los que conforman el consejo de administración se dediquen solo a tomar las decisiones de tipo empresarial. También hace mención de que no existe un reglamento fijo para la composición de un consejo familiar, pero que es recomendable que dentro del consejo participen representantes de distintas generaciones de la familia, es decir, que el consejo no solo sea de una sola generación, pero también lo deben integrar personas externas a la familia; estas personas deben tener experiencia empresarial y profesional.

Folle (2015) indica que existen 3 principales funciones de un consejo familiar, las cuales son:

1) Establecer y gestionar criterios, órganos y procedimientos

2) Cuidar de la familia y de las relaciones entre los miembros

3) Gestionar la información y la formación de los familiares

\section{Metodología}

Ce trata de un estudio descriptivo, con enfoque $\checkmark$ cuantitativo y de corte transversal, con una muestra representada por 11 participantes de empresas familiares ubicadas en Ciudad Juárez, Chihuahua. Para el levantamiento de datos se utilizó un cuestionario con diseño pro- pio, compuesto por 19 ítems, utilizando escala Likert de 5 puntos y preguntas dicotómicas, además de las variables demográficas. Este instrumento fue aplicado a propietarios o responsables de empresas familiares que hayan pasado por lo menos un proceso de sucesión. Los datos fueron analizados con el paquete estadístico SPSS versión 22.0.

\section{RESUltados}

e pudo observar que del
total de participantes, el
$46 \%$ de los sucesores de las empresas $(N=11)$ tiene una edad que oscila entre los 31 y 40 años, mientras que solo el $22 \%$ de ellos tiene entre 18 y 30 años. Lo anterior indica que la mayoría de los actuales dueños y/o propietarios se encuentra en una etapa de madurez. El $48 \%$ ha estado al frente de la empresa en un rango de 6 a 10 años, y solo un $8 \%$ tiene menos de un año. Solo el 8\% de las empresas encuestadas se encuentra en una tercera generación. En este caso el 100\% de los encuestados manifestó ser familiar del sucedido, siendo el 92\% hijo(a) del fundador.

A pregunta expresa sobre si hubo un conflicto al mo- 
mento de la sucesión, solo un $22 \%$ manifestó haberlo tenido, siendo con su misma familia. El 72\% manifestó tener conocimiento previo de que podría ser candidato a dirigir la empresa en algún momento; de igual manera, el $72 \%$ había ocupado algún cargo dentro de la empresa antes de ser seleccionado. El 92\% recibió con agrado la noticia de que sería seleccionado. Solo el 32\% ha hecho planes sobre cómo manejará la siguiente sucesión. El 82\% de la muestra manifestó conocer la misión y visión de la empresa.

Los primeros resultados indican que el $64 \%$ de los encuestados estuvo de acuerdo o totalmente de acuerdo en que recibió preparación especial, mientras que solo $18 \%$ estuvo en desacuerdo o totalmente en desacuerdo.

Sobre el impacto de la preparación de los sucesores, el $86 \%$ de los encuestados estuvo entre totalmente de acuerdo o de acuerdo en que la preparación recibida impactó positivamente, mientras que el $14 \%$ estuvo entre totalmente en desacuerdo o ni de acuerdo ni en desacuerdo.
De los encuestados, el 38\% estuvo ni de acuerdo ni en desacuerdo, mientras que el otro $62 \%$ se dijo de acuerdo o totalmente de acuerdo en que al momento de ocupar el puesto ya contaba con los conocimientos necesarios.

Por otra parte, el 62\% de los encuestados estuvo entre de acuerdo o totalmente de acuerdo en que sabían con anterioridad que existiría una vacante para ocupar el puesto, mientras que un $24 \%$ estuvo totalmente en desacuerdo

A la afirmación de si los encuestados participaron en un proceso de selección para ocupar la vacante, un $54 \%$ estuvo totalmente en desacuerdo, un $30 \%$ estuvo totalmente de acuerdo, mientras que solo el $16 \%$ estuvo ni de acuerdo ni en desacuerdo

Asimismo, el 38\% de los encuestados estuvo en desacuerdo o totalmente en desacuerdo en que los cargos de mayor importancia son ocupados por otros miembros de la familia, el 31\% estuvo ni de acuerdo ni en desacuerdo ante esta afirmación, el otro $31 \%$ dijo estar de acuerdo o totalmente de acuerdo.
De los encuestados, el 66\% estuvo totalmente de acuerdo en que el fundador o los antiguos jefes eran buenos líderes, mientras que solo un $18 \%$ estuvo en desacuerdo.

Al cuestionar sobre la influencia de los fundadores en la empresa, el 62\% de los encuestados estuvo totalmente en desacuerdo en que el antiguo líder o en su caso el fundador, aún tiene participación en las decisiones de la empresa, mientras que el $38 \%$ estuvo de acuerdo o totalmente en desacuerdo.

Así también, el 38\% de los participantes estuvo totalmente de acuerdo en que hizo cambios significativos en la política de la empresa, el 32\% estuvo ni de acuerdo ni en desacuerdo y solo el $7 \%$ estuvo totalmente en desacuerdo.

El 55\% de los empresarios estuvo totalmente de acuerdo en que durante su infancia se tocaron temas relacionados a la empresa en su hogar, mientras que solo el $15 \%$ estuvo totalmente en desacuerdo.

\section{Discusión}

Tomando en cuenta
las cuatro etapas a las
que hace referencia 
Modest (2012), se considera de suma importancia que con anticipación la familia involucrada en la empresa formule el perfil de quien podría ser el mejor y más adecuado candidato para ocupar el puesto principal en la empresa, considerando las características que debe tener el sucesor, identificando además si el prospecto necesita una preparación específica, ya sea profesional o en algún otro ámbito de personalidad, liderazgo, conocimientos, destrezas, etcétera, así como también familiarizarlo con los deberes diarios de la empresa, ya sea en el seno familiar o en la misma organización. Una vez que el sucesor sea nombrado oficialmente líder de la organización, toda la familia, incluido el sucedido, se encargarán de cobijarlo y aconsejarlo, buscando que se mantengan estables los intereses que a la familia convengan.

De acuerdo con Folle (2015), el compromiso del líder se basará en la identificación de este con los objetivos y valores aprendidos tanto en el hogar como en la empresa. Guerrero (2006) menciona que el desarrollo y fortalecimiento de estos valores será fundamental para el éxito de la empresa.

Modest (2000), Escobar (2014), Victor \& Jago (1990) coinciden en que la planeación es un aspecto fundamental para el éxito de la sucesión en una empresa familiar, y que si esta no se lleva a cabo de la forma correcta, será muy difícil que la organización sobreviva. Asimismo, el liderazgo es considerado por Gómez (2016) y Audisio (2014) como otro de los factores de gran peso para que la empresa continúe operando de manera satisfactoria.

\section{Conclusiones}

A partir de los resulta$A$ dos obtenidos, se pue$\mathcal{L}$ de determinar que la preparación del sucesor antes de ocupar el puesto es uno de los factores que influyen de manera importante. Se pudo observar que el 68\% de los encuestados manifestó haber recibido preparación especial antes de ocupar el puesto; $84 \%$ afirmó que la preparación recibida con anterioridad impactó positivamente al momento de quedar al frente de la empresa.

Otro de los aspectos destacables es la sucesión armoniosa, lo que demuestra el grado de confianza de que alguien de la familia se haga cargo directamente de la empresa. En ese sentido, el 78\% de ellos manifestó no haber tenido conflicto alguno al momento de tomar posesión de la empresa.

Otro aspecto importante a resaltar en esta investigación es que el $72 \%$ de los sucesores ya había ocupado algún puesto dentro de la empresa, así pues, se puede concluir que el conocimiento de la cultura y los valores que se han manejado en la empresa facilita el proceso de cambio en la organización, pues además se pudo observar que el $82 \%$ de los encuestados dijo conocer tanto la visión como la misión de su empresa.

El hogar formó también parte fundamental para lograr el compromiso y el sentido de pertenencia por parte del sucesor para el buen manejo de la empresa, el $55 \%$ de los encuestados estuvo totalmente de acuerdo en que durante su infancia se tocaron temas relacionados con la empresa en el seno familiar.

La imagen del sucedido también es un factor importante; en este estudio se pudo observar que el $66 \%$ de los participantes en la encuesta 
tiene una muy buena percepción del liderazgo ejercido por el encargado anterior.

Es de destacarse que de las encuestas realizadas, solo el $9 \%$ (1) fue de tercera generación, lo que confirma lo señalado por Álvarez (2014): solo el 35\% de las empresas familiares llegan a una segunda generación y de esas solo el $13 \%$ alcanza una tercera generación. El porcentaje es muy bajo y efectivamente aspectos como el liderazgo, el sentido de pertenencia y una buena planeación a largo plazo son factores que influyen para que las empresas familiares puedan subsistir.

\section{Bibliografía}

Álvarez, S. (2014). Expansión en alianza con CNN. Recuperado el 26 de enero de 2017, de http:// expansion.mx/emprendedores/2014/05/12/empresas-familiares-olvidan-sucesion

Arenas Cardona, H. A., \& Rico Balvin, D. (2014). La empresa familiar, el protocolo y la sucesión familiar. Estudios gerenciales, 255. Recuperado el 14 de marzo de 2017.

Audisio, N. (2014). La familia, una empresa hoy, mañana? (Primera ed.). Argentina: Brujas. Recuperado el 21 de febrero de 2017.

Camacho Bójorquez, J. E. (2012). Sucesión de liderazgo en organizaciones familiares grandes (Primera ed.). (S. Bañuelos, Ed.) Culiacán, Sinaloa, México: Ediciones del lirio S.A. de C.V. Recuperado el 21 de Marzo de 2017.

Cappuyns, K. (2008). El compromiso de los familiares con la empresa familiar. Universidad Autónoma de Barcelona, tesina inédita.

Chrisman, J. J., Chua, J. H., \& Sharma, P. (1998). Important atributies of succesors in family busines: an exploratory,. family business reviwe, 11. Recuperado el 11 de mayo de 2017.

Escobar, M. V. (2014). La sociedad por acciones simplificada como herramienta de planeación sucesoral. Revista de Derecho Privado. No. 51.

Folle, C. (2015). Compromiso y continuidad en las empresas familiares. IEEM Revista de Negocios, 54-59.

----(2015). El compromiso en la empresa familiar. IEEM revista de negocios, 8-9.

----(2015). El relacionamiento entre hermanos en la empresa familiar. IEEM Revista de Negocios, 8-10.

Gallo, M. A., \& Amat, J. M. (2003). Los secretos de las empresas familiares centenarias. Barcelona, España: Ediciones Deusto. Recuperado el 07 de Marzo de 2017.

García Álvarez, M. E. (2001). Los valores del fundador y su influencia en la empresa familiar en Galicia. Universidad Autónoma de Barcelo (tesis doctoral). Recuperado el 18 de mayo de 2017.

Gómez, G. (2016). Cómo realizar la sucesión del liderazgo empresarial familiar. IEEM Revista, (1), 28-32. 
Guerrero, V. S. (2006). Modelo explicativo de una empresa familiar que relaciona valores del fundador, cultura organizacional y orientación al mercado. Pensamiento y gestión, 194-215.

Lambrech, J., \& Astrachan, J. H. (1994). Influence of family relationships on succession planning and training. The importance of mediating factors. Family Business Review, 7. Recuperado el 11 de mayo de 2017.

Martínez Echezarraga, J. (2011). Empresas familiares, reto al destino: claves para perdurar con exito. Buenos Aires, Argentina: Ediciones Granica S-A-. Recuperado el 24 de abril de 2017.

Melquicedec, L. (2013). El proceso de socialización, vinculación y pertenencia de descendientes a la empresa familiar. Un estudio empírico en Colombia. Universidad Autónoma de Barcelona (Tesis publicaca en el 2013). Recuperado el 11 de mayo de 2017.

Modest, J. M. (2000). El empresario familiar y su plan de sucesión. Madrid, España: Díaz de Santos.

Press, E. (2011). Empresas de familia, del conflicto a la eficiencia (1a. ed.). Buenos Aires: Granica. Recuperado el 04 de abril de 2017.

Quijano García, R. A., Magaña Medina, D. E., \& Pérez Canul, C. A. (2011). Pertenencia y compromiso: factores relevantes en la transición gerencial de empresas familiares. Estudios Gerenciales, 99-113.

Roselia, A. M. (2016). La sucesión exitosa en las empresas familiares. Revista Orbis, 82-104. Recuperado el 09 de febrero de 2017.

Víctor H, V., \& Jago, A. G. (1990). El nuevo liderazgo, dirección de la participación en las organizaciones. Madrid: Díaz de Santos, S. A. Recuperado el 27 de Abril de 2017. 